\title{
Simulador multiciclo do processador MIPS 32 bits para apoio ao estudo em arquitetura de computadores
}

\author{
Warley Jacinto Elias - Faculdades Integradas de Caratinga - warleyelias@ gmail.com \\ Jacson Rodrigues Correia da Silva - Faculdades Integradas de Caratinga - \\ jacsonrcsilva@gmail.com
}

Fabrícia Pires Souza Tiola - Faculdades Integradas de Caratinga -

fabricia@doctum.edu.br

\section{Resumo:}

O uso de simuladores no contexto da educação vem se tornando uma estratégia cada vez mais adotadas na computação e outras ciências. Conteúdos complexos podem se tornar mais compreensíveis e mais interessantes ao serem praticados, vivenciados e experimentados. O Software Simulador da Arquitetura MIPS Multiciclo foi desenvolvido para auxiliar no ensino de Arquitetura de Computadores, buscando trazer maior absorção do conteúdo para os alunos e disponibilizar uma ferramenta de apoio para os professores. As avaliações realizadas com o uso do simulador demonstraram que a ferramenta é viável ao proporcionar aulas mais dinâmicas, intuitivas e atrativas, além disso o conhecimento em MIPS foi mais facilmente construído. Constatou-se que o Simulador estimulou os estudos e aumentou o interesse pelo conteúdo de Arquitetura de Computadores.

Palavras-chaves: Simulador, Arquitetura MIPS Multiciclo, Softwares Educacionais.

\section{Multicycle 32 bits MIPS processor simulator to support study in Computer Architecture}

\begin{abstract}
:
Simulators' use on educational context has become an strategy increasingly more adopted in computing and other sciences. Complex contents may become more understandable and more interesting when we practice, experience and try them. The Architecture Simulator Software (MIPS Multicicle) was developed to help Computers Architecture Teaching searching to bring up more content absorption by the students and turn available a supporting tool for teachers. The assessments performed with the use of Simulators show that the tool is viable providing more dynamic, intuitive and attractive classes. Besides that, knowledge on MIPS was easier constructed. It was confirmed that the Simulator boosted studies and increased the interest for computer architecture content.
\end{abstract}

Key-words: Simulator, Architecture MIPS Multicicle, Educational Softwares. 


\section{Introdução}

O ensino da arquitetura MIPS (Microprocessor Without Interlocked Pipeline Stages) na disciplina de Arquitetura de Computadores tem como objetivo introduzir o conceito do funcionamento dos processadores em geral. É utilizado por ser uma arquitetura simples, o que facilita na compreensão do caminho de dados e do controle de um processador. Este é um conteúdo um tanto complexo de se aprender, demandando um grande poder de abstração por parte dos alunos além de boa didática para os docentes.

Softwares educacionais são desenvolvidos para auxiliar alunos em várias áreas da computação e outras ciências. Estes softwares são cada vez mais sofisticados e intuitivos e tem por objetivo tentar levar para os alunos mais facilidades nos estudos dos conteúdos de disciplinas, ao proporcionarem testes e práticas de operações que não são facilmente realizadas sem o acesso físico a tais objetos.

Softwares Educacionais, segundo (Lucena, 1992), é todo aquele programa que possa ser usado para algum objetivo educacional, que seja pedagogicamente defensável por professores e alunos, ou que seja a natureza ou a finalidade para o qual tenha sido criado. No contexto do ensino de Arquitetura de Computadores, foram desenvolvidos e propostos vários simuladores cujo objetivo é permitir ao estudante, através de um software com uma interface gráfica visualizar e aprender os conceitos estudados. Dentre os simuladores existentes tem-se sistemas propostos por (Felix, 2006), (Barcellos, 2009), (Avelar, 2008), (Maia, 2009) e (Consendey, 2009).

Contudo há na Arquitetura de Computadores, assim como na computação em geral, áreas defasadas em material didático e em softwares para auxiliar no ensino das mesmas. Observando a carência de simuladores para as rotinas do processador MIPS, o objetivo deste trabalho foi desenvolver um software educacional capaz de simular as instruções assembly do MIPS Multiciclo, representando graficamente os componentes do caminho de dados e de controle.

Assim, foi implementado um software para auxiliar tanto os alunos quanto os professores para as aulas dessa disciplina. Com a utilização de simuladores, o aluno pode aprender desde conceitos básicos até tópicos mais avançados, conhecendo todos os componentes do processador da arquitetura MIPS e interagindo com cada um deles com mais naturalidade. $\mathrm{O}$ estudo em Arquitetura de Computadores pode se tornar mais fácil com o uso de Softwares Educacionais como simuladores, que permitem a abstração de detalhes do contexto apresentado.

\section{MIPS - PMS (MIPS Processor Multicycle Simulator)}

No mundo globalizado tem-se observado uma atuação cada vez maior dos computadores nas diversas atividades e no dia a dia das sociedades. As operações bancárias, as telecomunicações e o manuseio de muitos aparelhos eletrodomésticos são exemplos claros das facilidades fornecidas pela utilização dos computadores. Desde aplicações mais clássicas, como os sistemas de reservas de passagens aéreas e a previsão meteorológica, diversos setores da economia vem ao longo do tempo absorvendo e demandando o emprego de computadores.

Toda essa influência dos computadores alavanca a área da computação, como afirma (Patterson, 2005): "os computadores levaram a humanidade a enfrentar uma revolução, a revolução da informação, que assumiu seu lugar junto das revoluções 
industriais e agrícola". A evolução da informática foi caracterizada pelo desenvolvimento de computadores com características diversas, traduzidas pelos diferentes parâmetros, cada vez mais conhecidos da maioria de usuários de computador: a Unidade Central de Processamento $(C P U)$ adotada, a capacidade de memória, a capacidade do disco rígido, a existência de memória cache e outros menos conhecidos. A definição destes parâmetros e a forma como os diversos componentes de um computador são organizados, define aquilo que é conhecido por arquitetura de computador e vai determinar aspectos relacionados à qualidade, ao desempenho e à aplicação para a qual o computador orientar-se-á. Com essa grande ascensão tecnológica, a arquitetura de computadores também progride.

Este artigo aborda exclusivamente sobre a arquitetura da CPU MIPS Multiciclo, que possui todos os componentes básicos dos processadores e possui caminho de dados e controle mais simples que outras arquiteturas. Por ser menos complexo que os demais modelos de processador, os livros didáticos para o ensino da disciplina de Arquitetura de Computadores, utilizam esta arquitetura, um exemplo disso é (Patterson, 2005).

O software desenvolvido nesse trabalho foi denominado MIPS - PMS e simula exatamente o funcionamento do processador MIPS Multiciclo. Esse sistema computacional pode ser empregado pelo professor em suas aulas como complemento do material didático já utilizado.

O software pode ser empregado juntamente com a explicação teórica da arquitetura MIPS multiciclo, nas quais o professor pode apresentar como ocorre a execução desta arquitetura no simulador, mostrando, por exemplo, as mudanças nos valores dos registradores, tanto temporários quanto do banco de registradores, além de mudanças nos valores das posições de memória e as imagens do circuito, apresentando quais elementos estão sendo usados nas rotinas propostas.

O MIPS - PMS foi desenvolvido utilizando o framework NetBeans, que como consta em (Netbeansorg, 2010), é um ambiente de desenvolvimento integrado e multiplataforma, de código aberto e que contém uma plataforma de aplicativos que permite aos desenvolvedores criarem rapidamente aplicativos diversos utilizando diversas linguagens de programação. Foi utilizada também a plataforma Java, que é uma linguagem de programação orientada a objeto. A linguagem Java "possui versatilidade, eficiência, portabilidade de plataforma e segurança" (Java, 2010). A utilização do Java tem como objetivo atingir ao máximo de usuários possíveis utilizando a portabilidade que a mesma possui, assim, pode ser executado em diversos sistemas operacionais, como Microsoft Windows, Mac OS e derivados do Linux. No desenvolvimento do simulador, a codificação foi realizada de forma padronizada, facilitando assim a modificação do código-fonte para possíveis atualizações e melhorias no simulador.

A interface do sistema foi criada para ser intuitiva, limpa e dinâmica, contendo apenas os campos e botões necessários para executar os códigos assembler do processador MIPS referenciados em (Patterson, 2005) de maneira eficaz e simples. A simplicidade na interface teve por objetivo não dispersar a atenção dos alunos na hora da usar do simulador, focando nas mudanças causadas pela execução das instruções na interface. Isso pode ajudar mais os alunos a entender o funcionamento da arquitetura.

Quanto a sintática do código assembler, a utilização se restringe em utilizar espaços entre cada item da instrução, a sintática utilizada nas instruções foi baseada no livro de Patterson (2005), com intuito de que o aluno possa utilizar os conceitos teóricos da sintaxe das instruções também no simulador, facilitando assim o emprego do mesmo. 
Na sequência do texto está um exemplo da especificação da sintaxe correta de cada instrução que o simulador reconhece:

- addi \$1 \$1 10;

- $\quad$ add $\$ 3 \$ 1 \$ 2$;

- $\quad$ subi $\$ 4 \$ 33$;

- $\quad$ sub $\$ 5 \$ 3 \$ 1$;

- $\quad$ and $\$ 6 \$ 2 \$ 5$;

- $\quad$ or $\$ 7 \$ 1 \$ 2$;

- $\quad$ xor $\$ 8 \$ 5 \$ 4$;

- $\quad$ sw $\$ 3 \$ 0$ ( 204 );

- $\quad$ lw $\$ 10 \$ 0$ ( 204 ).

A Figura 1 mostra o layout do simulador MIPS - PMS que contém 3 painéis: um painel com os registradores, onde é possível analisar os valores presentes nos 32 registradores; um painel de registradores temporários, com os valores presentes em cada um dos referidos registradores temporários; e um painel que contém todas as posições de memórias, mostrando os valores na parte de dados da memória e mostrando também a parte onde ficam as instruções. 


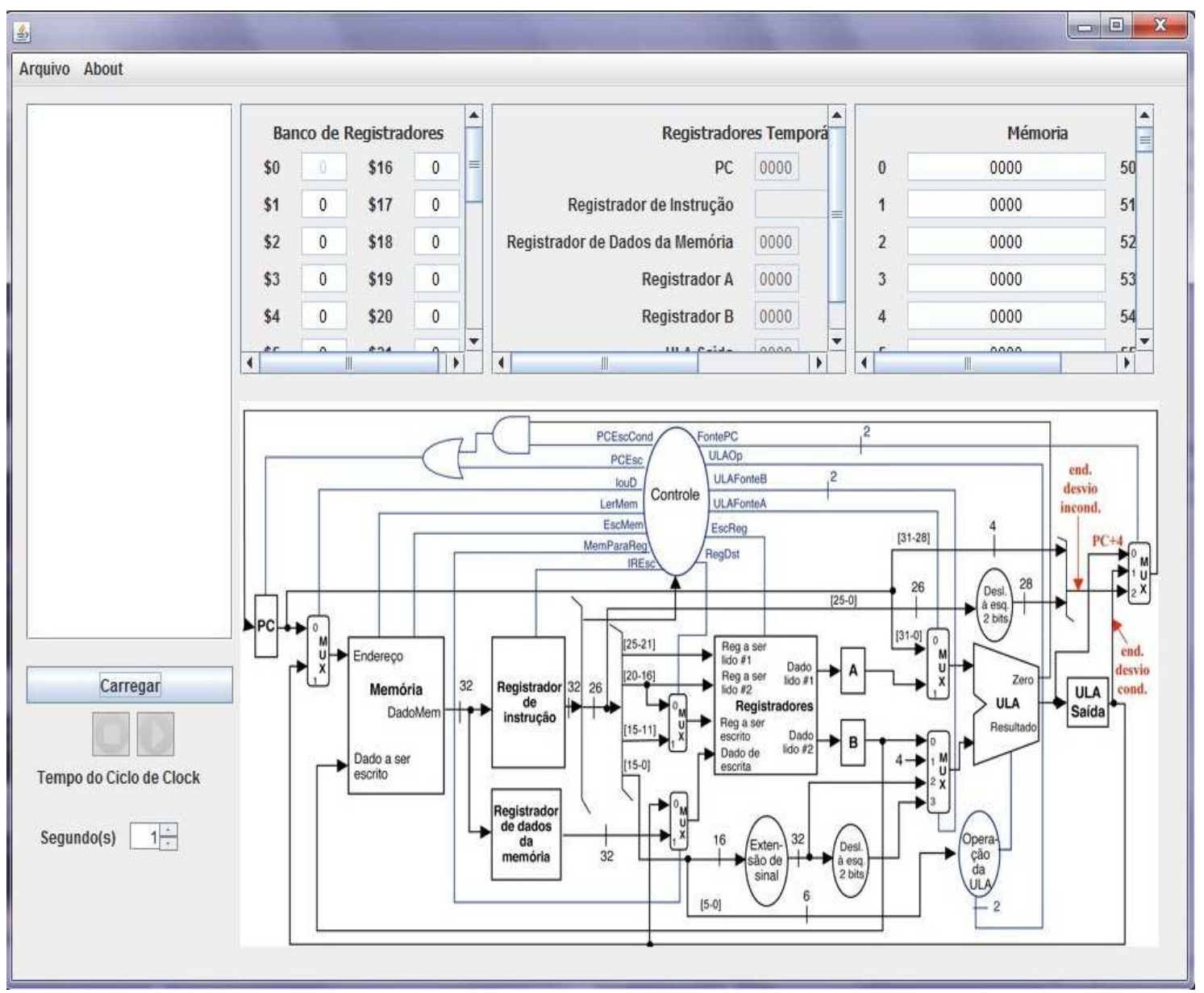

Figura 1 - Layout do simulador MIPS - PMS

Na Figura 1 é apresentada a interface do software desenvolvido. A imagem do circuito é atualizada a cada passo de execução das instruções do processador MIPS, mostrando os componentes utilizados em cada etapa da instrução para exemplificar o que ocorre no caminho de dados.

O programa conta com um campo de texto para inserir o código ou este pode ser carregado ou salvo em um arquivo por meio do menu Arquivo. O simulador ainda conta com um campo texto, demonstrado também na Figura 1, onde é possível digitar um código assembler, respeitando as instruções que o programa reconhece e suas sintáticas, para que o aplicativo possa executá-las. Duas opções para tratar desse código também estão presentes no simulador: a opção de salvar o código digitado, onde o usuário pode salvar o código em um arquivo para futuras execuções; e a opção de carregar um código já existente, que carrega o arquivo salvo para o campo texto do simulador e permite também ao usuário alterar o código carregado.

Um painel contendo apenas a imagem representativa do circuito da arquitetura, MIPS Multiciclo foi adicionado ao simulador como apresentado na Figura 1. Esta serve para atualizar a imagem de acordo com a instrução que está sendo executada e de acordo com cada passo de sua execução, identificando quais componentes do circuito estão sendo executados e os 7valores de controle que estão sendo utilizados pela instrução em execução.

As cores Preta, Vermelha e Azul na imagem servem para diferenciar elementos e processos. O Preto é o caminho de dados, por onde passam os dados para serem 
utilizados na execução do ciclo de clock. Em Azul, estão os sinais de controle, que são utilizados para definir o que cada componente do caminho de dados realiza a cada ciclo. Já em Vermelho, são destacados alguns dados importantes que estão passando pelo caminho de dados.

Na parte inferior esquerda da Figura 1 observa-se os botões "play", "stop" e "carregar", além do "contador de segundos", que determina a velocidade de cada clock. O funcionamento destes elementos será explicado à seguir. Os botões de controle e execução, que podem ser utilizados para carregar, dar início, ou parar a execução do código foram criados com intuito de fornecer o controle da execução das instruções aos usuários. Ao carregar o código, o simulador faz uma análise sintática do mesmo, essa funcionalidade foi desenvolvida para mostrar aos usuários como é a sintática das instruções, para que os mesmos entendam também o que cada parte da instrução representa para sua execução.

O software possui um campo no qual se pode regular a velocidade (em segundos) dos ciclos de clock para que se ajustem a velocidade da execução da simulação, tudo isso para que possam adequar à simulação e acompanhar a execução da melhor forma possível. Ele também abriga um painel que fornece as informações sobre todos os trinta e dois registradores que compõe esta arquitetura. Como já foi citado, este painel é atualizado a cada ciclo de clock, mostrando ao usuário como determinadas instruções mudam os valores destes registradores em cada passo de sua execução. $\mathrm{O}$ intuito da criação deste painel foi deixar claro para o usuário do simulador o conteúdo presente no banco de registradores, bem como as modificações realizadas pelas instruções nos valores presentes nesse banco de registradores. A melhor visualização deste painel está na Figura 3, na parte esquerda.

Há ainda outro painel que mostra os registradores de estado, onde ficam as informações que serão utilizadas de um ciclo para outro subseqüente. Este painel foi criado com a intenção de mostrar para o usuário quais valores necessitam ser salvos nesses registradores temporários para que o próximo ciclo de clock não seja perdido no meio da simulação (vide Figura 3).

Outro painel importante é o que apresenta os valores contidos na memória. Essa memória é dividida para guardar tanto instruções quanto dados. Este painel atualiza a cada ciclo de clock seus valores e foi criado com objetivo de mostrar para o usuário do simulador como as instruções ficam salvas na memória e como essa mesma é sua divisão para salvar tanto instruções quanto dados. Além disso, o painel também foi criado com intuito de mostrar a dinâmica de acesso à memória, para que o usuário consiga ver e compreender os locais onde os dados podem ser salvos e onde fica a parte das instruções que ficam bloqueadas para o usuário (vide Figura 3, na parte direita).

\begin{tabular}{|c|c|c|c|c|c|c|c|c|c|c|}
\hline \multicolumn{4}{|c|}{ Banco de Registradores } & - & \multicolumn{3}{|c|}{ Registradores Temporá } & \multirow[b]{2}{*}{0} & \multirow{2}{*}{$\begin{array}{l}\text { Mémoria } \\
\text { addi } \$ 1 \$ 110\end{array}$} & \multirow{2}{*}{$\underset{50}{\underline{\Delta}}$} \\
\hline$\$ 0$ & 0 & $\$ 16$ & 0 & $=$ & PC & 0000 & & & & \\
\hline$\$ 1$ & 0 & $\$ 17$ & 0 & - & Registrador de Instrução & & & & 0 & 51 \\
\hline$\$ 2$ & 0 & $\$ 18$ & 0 & & Registrador de Dados da Memória & 0000 & & & 0 & 52 \\
\hline$\$ 3$ & 0 & $\$ 19$ & 0 & & Registrador A & 0000 & & & 0 & 53 \\
\hline$\$ 4$ & 0 & $\$ 20$ & 0 & & Registrador B & 0000 & & & 0 & 54 \\
\hline 4 & & a & $-a$ & $\vec{v}$ & 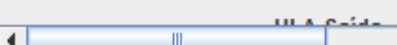 & ممممه & A & 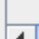 & & $=7$ \\
\hline
\end{tabular}

Figura 2 - Painéis que contém os valores dos registradores temporários, banco de registradores e memória presentes no layout do simulador MIPS - PMS. 
A barra de rolagem deve ser utilizada para acessar os elementos presentes na interface, que ficaria muito carregada se fosse aplicado todas estas informações em seu layout final como apresentado na Figura 2.

\section{Resultados}

Para avaliar a viabilidade de se utilizar o Simulador MIPS-PMS em disciplinas de Arquitetura de Computadores, foi realizada uma pesquisa, junto à comunidade acadêmica de um determinado curso de Ciência da Computação. Os alunos da disciplina foram treinados a utilizarem o sistema após ser lecionado de modo tradicional todo o conteúdo sobre os processadores MIPS e o seu funcionamento, ou seja, os acadêmicos conheciam o funcionamento do processador que foi apresentado por meio de exposição oral pelo docente da disciplina, sendo também realizadas atividades para fixação do conteúdo.

No total 15 alunos do curso foram treinados a manusear o Simulador, executar testes de diversas instruções e após se familiarizarem com o sistema, responderam 12 à questões relativas ao uso do software como apoio na disciplina de Arquitetura de Computadores. As questões elaboradas seguem na Tabela 1.

Tabela 1 - Questões utilizadas no questionário.

\begin{tabular}{|l|l|}
\hline \multicolumn{2}{|c|}{ Questões } \\
\hline Questão 1 & Qual o grau de facilidade em utilizar o simulador MIPS - PMS? \\
\hline Questão 2 & $\begin{array}{l}\text { Qual o grau de facilidade em acompanhar a execução das instruções no } \\
\text { simulador? }\end{array}$ \\
\hline Questão 3 & O simulador trouxe facilidade na compreensão do conteúdo? \\
\hline Questão 4 & A interface é intuitiva? \\
\hline Questão 5 & A interface é agradável? \\
\hline Questão 6 & $\begin{array}{l}\text { A interface é simples e contém o conteúdo principal para visualizar a } \\
\text { execução das instruções? }\end{array}$ \\
\hline Questão 7 & $\begin{array}{l}\text { Você utilizaria esse simulador como complemento para o estudo em } \\
\text { Arquitetura de Computadores? }\end{array}$ \\
\hline Questão 8 & $\begin{array}{l}\text { A funcionalidade de abrir um arquivo contendo o código assembler } \\
\text { facilita a utilização do simulador? }\end{array}$ \\
\hline
\end{tabular}




\begin{tabular}{|l|l|} 
Questão 9 & $\begin{array}{l}\text { A funcionalidade de salvar um arquivo contendo o código assembler } \\
\text { facilita a utilização do simulador? }\end{array}$ \\
\hline Questão 10 & Qual o grau de satisfação em utilizar o simulador? \\
\hline Questão 11 & Qual seu grau de interesse em Arquitetura de Computadores? \\
\hline Questão 12 & $\begin{array}{l}\text { Qual seu grau de interesse em estudar a arquitetura dos processadores } \\
\text { MIPS? }\end{array}$ \\
\hline
\end{tabular}

A Tabela 1 contém as perguntas que foram utilizadas no questionário de avaliação do simulador MIPS - PMS. Era possível se assinalar Péssimo, Ruim, Regular Bom ou Ótimo, sendo atribuído os valores de 1 a 5 respectivamente para cada alternativa. Somente a questão 7 era possível assinalar Sim ou Não. Este questionário foi respondido após os alunos executarem uma atividade, nas quais o simulador deveria ser manipulado individualmente e sem o auxílio de monitores ou tutores.

Foi gerada uma tabela de estratificação com as porcentagens das respostas assinaladas. A Tabela 2 apresenta os resultados obtidos em cada questão respondida pelos 15 alunos do curso de Arquitetura de Computadores.

Tabela 2 - Resultado da Avaliação.

\begin{tabular}{|c|c|c|c|c|c|c|}
\hline & Questão 1 & Questão 2 & Questão 3 & Questão 4 & Questão 5 & Questão 6 \\
\hline 1 (Péssimo) & $0,00 \%$ & $0,00 \%$ & $0,00 \%$ & $0,00 \%$ & $0,00 \%$ & $0,00 \%$ \\
\hline 2 (Ruim) & $0,00 \%$ & $0,00 \%$ & $0,00 \%$ & $6,67 \%$ & $0,00 \%$ & $0,00 \%$ \\
\hline 3 (Regular) & $6,67 \%$ & $13,33 \%$ & $13,33 \%$ & $13,33 \%$ & $13,33 \%$ & $0,00 \%$ \\
\hline 4 (Bom) & $46,67 \%$ & $46,67 \%$ & $53,33 \%$ & $26,67 \%$ & $53,33 \%$ & $40,00 \%$ \\
\hline \multirow[t]{2}{*}{5 (Ótimo) } & $46,67 \%$ & $40,00 \%$ & $33,33 \%$ & $53,33 \%$ & $33,33 \%$ & $60,00 \%$ \\
\hline & Questão 8 & Questão 9 & Questão 10 & Questão 11 & Questão 12 & \\
\hline 1 (Péssimo) & $0,00 \%$ & $0,00 \%$ & $0,00 \%$ & $6,67 \%$ & $13,33 \%$ & \\
\hline 2 (Ruim) & $0,00 \%$ & $0,00 \%$ & $0,00 \%$ & $0,00 \%$ & $0,00 \%$ & \\
\hline 3 (Regular) & $20,00 \%$ & $13,33 \%$ & $6,67 \%$ & $26,67 \%$ & $13,33 \%$ & \\
\hline 4 (Bom) & $40,00 \%$ & $46,67 \%$ & $53,33 \%$ & $40,00 \%$ & $66,67 \%$ & \\
\hline
\end{tabular}




\begin{tabular}{|l|c|c|c|c|c|}
5 (Ótimo) & $40,00 \%$ & $40,00 \%$ & $40,00 \%$ & $26,67 \%$ & $6,67 \%$ \\
\hline & Questão 7 & \multicolumn{3}{|c}{} \\
\cline { 1 - 2 } Sim & $100,00 \%$ & & & \\
\cline { 1 - 2 } Não & $0,00 \%$ & & &
\end{tabular}

Os números mostrados na Tabela 2 expõem a avaliação dos alunos sobre o simulador, onde os mesmos avaliaram as funcionalidades, interface, facilidade na compreensão da execução, na utilização e interesse na matéria de Arquitetura de Computadores. Acredita-se que se o aluno não se interessa pelo conteúdo, certamente o simulador não trará muitos rendimentos ao acadêmico.

Analisando os resultados, pode-se observar que na maioria das questões os alunos aprovaram o simulador e suas funcionalidades, mas faz-se uma ressalva que o mesmo ainda precisa de algumas modificações para ser melhor avaliado. Para a melhor análise dos resultados, foi gerado um gráfico com as questões propostas. A Figura 3 expõe o gráfico gerado e a figura 4 apresenta o gráfico referente a Questão 7.

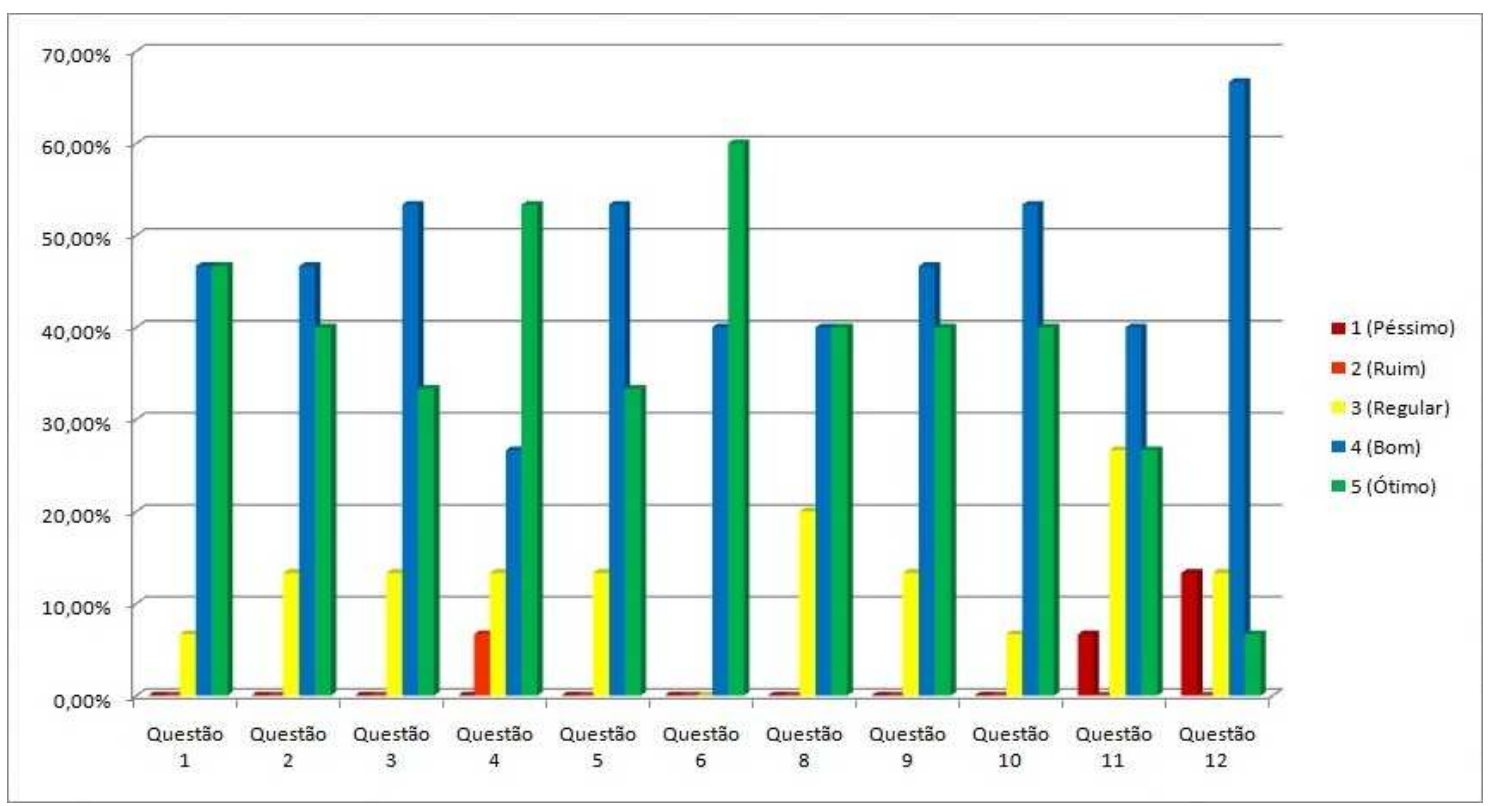

Figura 3 - Gráfico com o resultado do questionário.

A Figura 3 apresenta como as funcionalidades do Simulador foram avaliadas pelos alunos, demonstrando que no geral o simulador foi avaliado positivamente, possuindo uma boa interatividade, facilidade de uso e compreensão do conteúdo. A grande maioria assinalou as opções Ótimo ou Bom para tais aspectos da interface (vide questões 1 a 6 ).

As funcionalidades Abrir e Salvar, presentes na parte inferior esquerda da Figura 1, apresentaram algumas avaliações Regulares, mas as opções Ótimo e Bom predominaram na opinião dos usuários que testaram o Software.

O grau de satisfação ao se utilizar o Simulador foi bem alto, pois também se predominaram as opções Ótimo e Bom. Já nas questões 11 e 12 ao se arguir sobre o interesse na área de Arquitetura de Computadores e nos processadores MIPS foi constatado que alguns alunos têm pouco interesse nos referidos temas.

Finalmente, para descobrir se o Simulador seria empregado para o estudo dos acadêmicos, como um complemento no material didático-pedagógico, $100 \%$ dos alunos 
declararam que SIM, utilizariam o Software para apoiar no aprendizado de Arquitetura de Computadores. Vide respostas no gráfico da Figura 4.

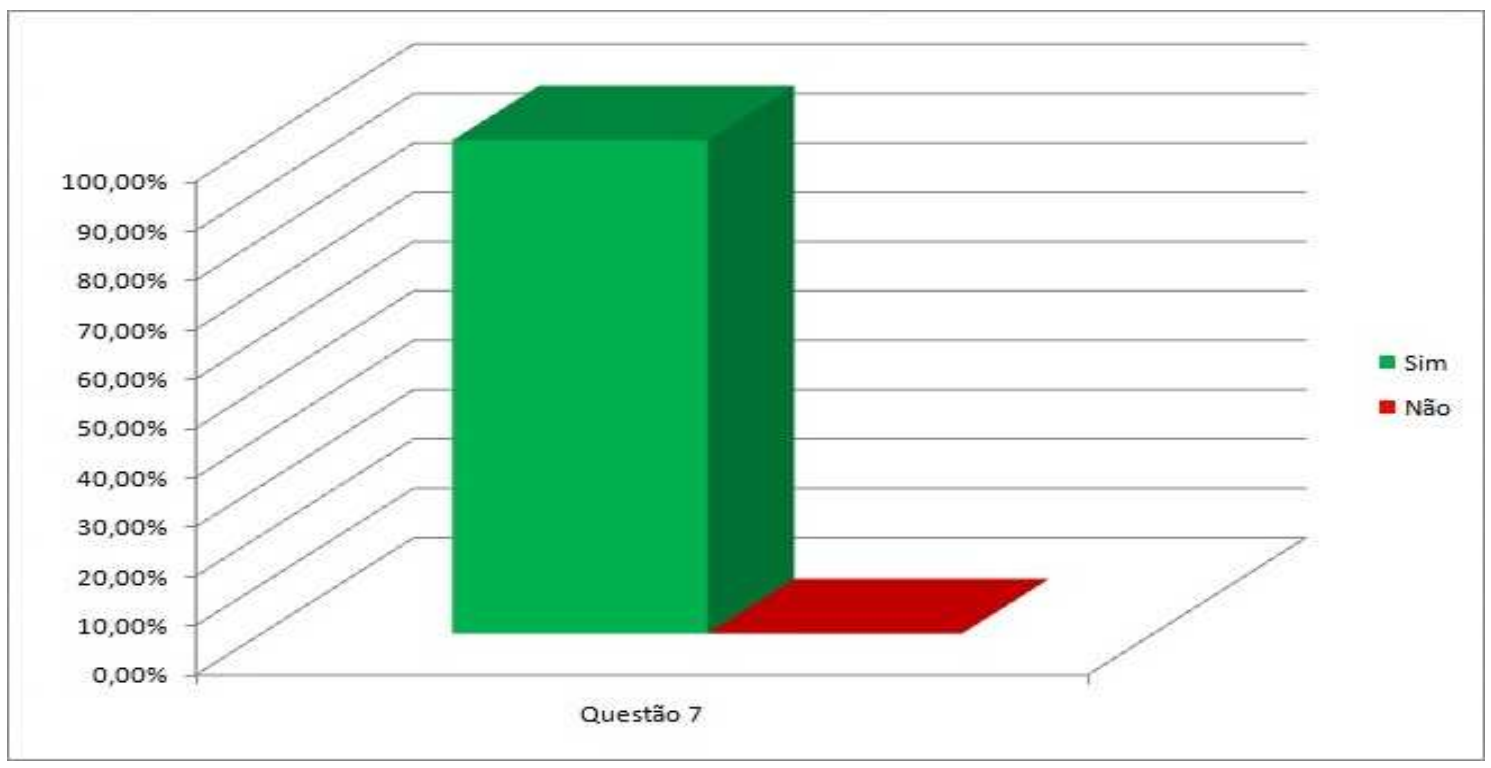

Figura 4 - Você utilizaria esse simulador como complemento para o estudo em Arquitetura de Computadores?

Na Figura 4, que apresenta o resultado da Questão 7 (Tabela 1), nota-se que $100 \%$ dos alunos entrevistados afirmaram que gostariam de utilizar o simulador MIPS PMS para complementar o estudo em Arquitetura de Computadores.

A maioria das respostas foram positivas. O ponto que deve ser modificado em trabalhos futuros é em questão da adição de mais painéis contendo o conteúdo de outros componentes da arquitetura dos processadores MIPS Multiciclo para que o programa se torne ainda mais intuitivo.

\section{Conclusão}

O aprendizado de um conteúdo abstrato e de difícil acompanhamento de passos e processos pode ser facilitado com o uso de simuladores. A interatividade nas aulas e praticidade sempre são aspectos muito desejados por todos os estudantes que esperam aulas mais dinâmicas e intuitivas. A criação do simulador MIPS - PMS é uma iniciativa de se promover o estudo de Arquitetura de Computadores de modo mais atraente e prático para alunos e professores. A interatividade promovida com o Simulador MIPSPMS facilita a compreensão do conteúdo e pode atrair a atenção do aluno, por propiciar na prática as instruções e operações realizadas de forma intuitiva e sequencial.

Com a pesquisa de aceitação do Simulador, pode-se perceber que o mesmo foi bem aceito pelos alunos entrevistados, alcançando o objetivo de ser utilizado como apoio no estudo da disciplina de Arquitetura de Computadores. Espera-se que além disso o Simulador MIPS-PMS possa motivar aos alunos que não possuem interesse na disciplina de Arquitetura de Computadores, facilitando a compreensão de um conteúdo que é básico no ensino da Computação.

De modo geral, foi verificado que a aceitação do simulador MIPS-PMS foi grande, e que se faz necessário a criação de novos simuladores. Este pode ser 
distribuído aos interessados e também melhorado, aumentando assim a gama de softwares educacionais para o ensino da Computação.

\section{Referências}

AVELAR, Cíntia P.; et al. MPDSim: Simulador didático do Pipeline do MIPS de 32 bits. In: Workshop sobre Educação em Arquitetura de Computadores (WEAC), 2008, Campo Grande.

BARCELLOS, Cézar da Cunha; SIMÕES, Henrique Magalhães. Ultimate Pipeline Simulator: Simulador do Pipeline do MIPS. In: Workshop sobre Educação em Arquitetura de Computadores (WEAC), 2009, São Paulo.

CONSENDEY, Michael Martins; BRASIL, Rafael Henrique; MARTINS, Carlos A. Paiva da Silva. SIPPIS MIPS: Um Novo Simulador de Pipeline do MIPS 32 bits para Auxílio à Educação em Arquitetura de Computadores. In: Workshop sobre Educação em Arquitetura de Computadores (WEAC), 2009, São Paulo.

FELIX, Ariane F.; POUSA, Christiane V.; CARVALHO, Milene B. DIMIPSS: Um simulador didático e interativo do MIPS. In: Workshop sobre Educação em Arquitetura de Computadores (WEAC), 2006, Ouro Preto.

JAVA - Saiba mais sobre a tecnologia Java. Disponível em: <http://www.java.com/pt_BR/about/>. Acessado em: 05 de out. 2010.

LUCENA, M. A Gente é uma Pesquisa: Desenvolvimento Cooperativo da Escrita Apoiado pelo Computador; Dissertação de Mestrado; Departamento de Educação, PUC - Rio; Rio de Janeiro: 1992.

NETBEANSORG - NetBeans Org. Disponivel em: $<$ http://netbeans.org/community/releases/69/index_pt_BR.html>. Acessado em: 05 de out. 2010.

PATTERSON, D. A.; HENNESSY J. L. Organização e Projeto de Computadores A Interface Hardware/Software. $3^{\text {a }}$ Edição, Editora Campus, 2005. 